\title{
General Relativistic Gravity Machine using Electromagneto-Torsion Field
}

\section{Takaaki Musha1 ${ }^{1}$, Mario J.Pinheiro²}

\author{
${ }^{1}$ Advanced Sci.-Tech. Research Organization, Yokohama, Japan \\ ${ }^{2}$ Dept. of Physics, Instituto Superior Técnico, Universidade de Lisboa, Lisbon, Portugal
}

\begin{abstract}
Some field propulsion systems are based on the solution of General Relativity Theory and are related to the connection between gravity and electromagnetic field. For example, Robert Forward proposed a gravity machine working according to the Einstein's General Relativity Theory, which requires ultra dense matter with the density of a dwarf star to produce useful thrust, but the proposed theoretical scheme cannot be realized by conventional technologies. The authors propose several concepts of a gravity machine utilizing an intense electromagnetic field that produces sufficient thrust to propel the spaceship, in accordance with Einstein's General Relativity Theory.
\end{abstract}

\section{Introduction}

A breakthrough propulsion method has been required for the purposes of interplanetary and interstellar travel. Instead of conventional chemical propulsion systems, field propulsion systems, which are based on the General Relativity Theory, have been proposed by many researchers. Some of them are based on solutions of the General Relativity Theory and are related to a connection between gravity and electromagnetic field. The Unified Field Theory on the connection between gravity and electromagnetic field was obscure until the present time.

On the other hand, Robert L. Forward described the theoretical generation of dipole gravitational fields by accelerating a super-dense fluid through pipes wound around a torus. Such techniques, though theoretically sound, have been far beyond the reach of current technology [1-8]. In his gravity machine, a mass flow through a pipe wound around a torus produces a co-gravitational field in a torus. If the mass flow is accelerated, the co-gravitational field increases with time and a dipole gravitational field is created. If the pipe is filled with a massive liquid and this liquid is moved back and forth in the pipe rapidly enough, then an alternating push-pull gravity field will be generated at the center of the ring. If the machine has the appropriate scale, the liquid is dense enough and moves fast enough, we have a gravity catapult that could launch and retrieve spaceships by using its gravity repulsion and attraction. An appropriate configuration should have the form of a ring of ultra-dense matter (the density of a dwarf star) and using this a flying body could be expelled out the other side of the hole with a greatly increased velocity. But this system is far beyond the conventional technologies and so the authors proposed a system which generates a thrust by an intense electromagnetic field inside a torus instead of an ultra-dense material. This electromagnetic method is in accordance with Einstein's general relativity theory.

\section{Forward's Gravity Machine}

It is known that there is an analogical relation between electromagnetic field and gravitational field documented in research papers by Harris and Braginsky $[9,10]$ and shown as follows.

A particle mass $m$ moving in a gravitational and electromagnetic field follows its path according to the equation of motion

$$
\frac{d^{2} x^{\mu}}{d \tau^{2}}+\Gamma_{\alpha \beta}^{\mu}\left(\frac{d x^{\alpha}}{d \tau}\right) \cdot\left(\frac{d x^{\beta}}{d \tau}\right)=\frac{e}{m c} F_{v}^{\mu}\left(\frac{d x^{y}}{d \tau}\right),
$$

where $e$ is the charge of a mass $\mathrm{m}$, $\Gamma_{\alpha \beta}^{\mu}=g^{\mu \sigma}\left(\partial_{\alpha} g_{\sigma \beta}+\partial_{\beta} g_{\alpha \sigma}-\partial_{\sigma} g_{\alpha \beta}\right) / 2$ are Christoffel symbols of the second kind and $F^{\mu v}$ is the electromagnetic field tensor. The Einstein's field equation of gravitation is given by

$$
R_{\mu \nu}=-\frac{8 \pi G}{c^{4}}\left(T_{\mu \nu}-\frac{1}{2} g_{\mu \nu} T\right)
$$


From which, Harris obtain equations for the case when the particle is slowly moving compared to the speed of light and the gravitational field is sufficiently weak so that nonlinear terms in Einstein's field equations can be neglected-Harris [9] obtained the following set of magnetic-type gravity equations

$$
\begin{aligned}
& \nabla \cdot g=-4 \pi G \rho, \\
& \nabla \cdot K=0, \\
& \nabla \times g=0 \quad\left(\nabla \times g=-\frac{\partial K}{\partial t}\right), \\
& \nabla \times K=-\left(\frac{4 \pi}{c}\right) \cdot(4 G \rho v)+\frac{4}{c} \frac{\partial g}{\partial t}, \\
& F=e\left(E+\frac{v \times B}{c}\right)+m\left(g+\frac{v \times K}{c}\right)+\frac{m v}{2 c} \frac{\partial \varphi}{\partial t}
\end{aligned}
$$

where $G$ is the gravitational constant, $g$ is the gravitational field, $K$ is the co-gravitational field (or gravito-magnetic field), $\rho$ is the mass density, and $\varphi$ is the scalar potential of electromagnetic field. One of the most remarkable attribute of the general theory of relativity is to predict the existence of gravitational effects analogous to the magnetic effects of electromagnetism.

By using the analogy to electromagnetism, the so-called gravitomagnetic gravitational field, having momentum of moving bodies as its source, Forward has shown in his paper that it is possible to build a machine to create a gravitational field using a system of accelerated masses [6]. To bypass the complex procedure of handling tensor algebra, lengthy and not always leading to physically observable effects, Forward proposed a set of analogues physical quantities from where he obtained useful connections. As it is well-known, a time varying magnetic field creates a dipole field, and the value of the electric field at the center of the torus is

$$
E=-\dot{B}=\frac{d}{d t} \frac{\mu N I r^{2}}{4 \pi R^{2}},
$$

where $R$ is the radius of the torus, $r$ is the radius of one of the loops of wire wound around it and $N$ is the total number of turns.

If we replace the wires with pipes carrying a massive liquid, then the known analogy between the electromagnetic and gravitational field can be applied. Then the equivalent gravitational quantities can be given by

$$
g=-\dot{K}=-\frac{d}{d t}\left(\frac{\eta N T r^{2}}{4 \pi R^{2}}\right),
$$

where $g$ is the gravitational field generated by the total accelerated mass, as shown in Fig. 1.

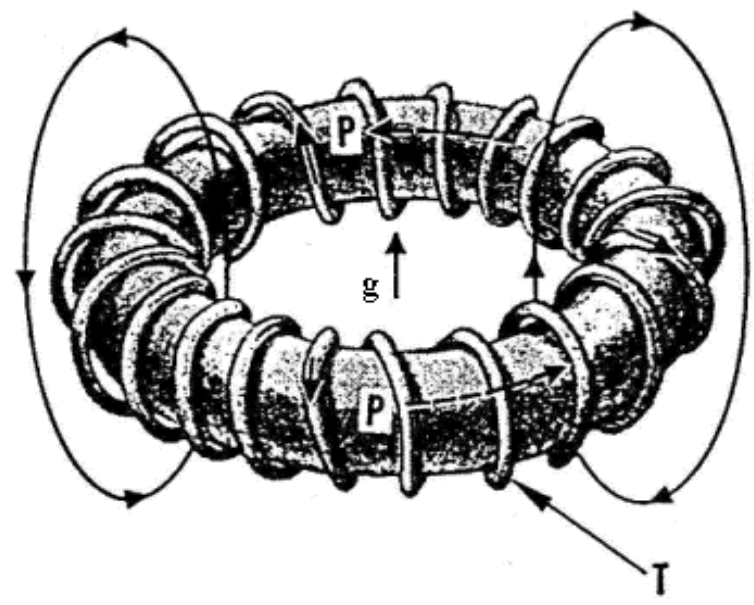

Figure.1 Generator of a dipole gravitational field

Supposing that the gravitational permeability has the value $\eta=3.73 \times 10^{-26} \mathrm{~m} / \mathrm{kg}$, we have $g \approx 10^{-10} a$ ( $a$ : amount of acceleration) at the center of the torus, as shown in Fig.5. This is for the case when the mass of the liquid through the pipes has the density of a dwarf star, the pipes are as wide as a football field and are wound around a torus with kilometer dimensions, according to Forward, which is far from present engineering capabilities.



Figure.2 Gravitational machine according to Forward's idea

By utilizing this gravitational machine, constant upward gravitational field can be generated which 
can be used as a gravitational catapult capable of pushing a body, as shown within the center of the torus, in Fig.2.

Instead of this gravity machine, which cannot actually be built, another gravity machine utilizing an intense electromagnetic field can be considered.

\section{Gravitational Machine by utilizing the Electromagneto-Torsion Field}

As early as 1951, P.A.M. Dirac published two papers where he pointed out that we should take into account quantum fluctuations in the flow of the aether $[12,13]$. Inspired by the Dirac ideas, K.P. Sinha, C. Sivaram and E.C.G. Sudarshan published in 1975 a series of papers that suggested a new model for the aether, in which it is a superfluid state of fermion and anti-fermion pairs, describable by a macroscopic wave function [14-16]. In their papers, they decided to treat the superfluid as relativistic matter - by putting it into the stress-energy tensor of the Einstein field equations. This allows us to take an important step - allowing us to describe relativistic gravity as one of the small fluctuations of the superfluid vacuum as well. Based on their ideas, we can consider the possibility of a space propulsion utilizing the co-gravitational field from the standpoint of hydrodynamics. Such a term implies various fluid dynamics which includes linear flow, separated flow as well as a creation of vortices.

\subsection{Theory of the Electromagneto-Toroidal Structure}

Among the various processes used in the natural world, there is a common principle that relies on the production of vortices by a material structure [17] (e.g., wings, paddles, fins). Most probably this fact is the realization of a general law of nature, to which is associated we may call a local "twist" of the fluid, firstly investigated by Viktor Schauberger and Professor Von Karman with his experiments with the flow for some distance behind a plate showing a regular arrangement of vortex lines, the so called "vortex street", that remain behind the plate and advancing at a more slow pace than the plate itself. The observation of these facts make us to turn our attention to the problem of the electrodynamic acceleration of fluids by reaction against the physical vacuum [18-23], or plasma medium, or any other kind of fluid [17].

Our purpose in this section is to show that Magnus and Abraham forces are a mathematical representation of the same type of structure in the fabric of space-time: a vortex capable of propelling material bodies.
To make the idea of this underlying principle so much simple, we may start to recall that, in the framework of electrodynamics the ponderomotive force acting on the material of an electromagnetic propulsion device is provided by Abraham's force density, $\vec{f}_{A}$, a term which represents the force communicated to a material structure (e.g., Refs. [20,22-29]). It is also known that, in the framework of hydrodynamics, the three-dimensional Magnus force is given by [28]:

$\vec{f}_{M x y}=-\rho\left[\left(\vec{V}_{v}-\vec{V}_{v o}\right) \times \vec{k}_{z}\right]$

where $\vec{V}_{v 0}$ is the velocity of the vortex center of mass, $\vec{V}_{v}$ is the stream velocity, $\rho$ is the fluid density, and $\vec{k}_{z}$ is a vector oriented along the $z$ direction with magnitude equal to the circulation. We may notice now that the "magnetic current force" created by the magnetic charges that "flow" when a magnetic field changes, is given by $\vec{f}_{m}=\varepsilon_{0}\left[\vec{E} \times\left(\dot{\vec{B}}-\mu_{0} \dot{\vec{H}}\right)\right]$ (see discussion of the concept in Ref. [27]). This is the "Abraham term" appearing in the Abraham force density $\vec{f}_{A}$, which differs from the Minkowski force density by means of the expression (see also Refs. [24,29] for further clarification):

$\vec{f}_{A}=\vec{f}_{M}+\frac{\partial}{\partial t}\left(\frac{\varepsilon_{r} \mu_{r}-1}{c^{2}}[\vec{E} \times \vec{H}]\right)$

The last term of Eq.11 corresponds to the vacuum-interactance, a concept introduced in Ref. [30], and meaning a process of pushing material bodies through interaction with the physical vacuum, which is associated with the momentum as follows:

$\vec{g}^{V}=\frac{\varepsilon_{r} \mu_{r}-1}{c^{2}}[\vec{E} \times \vec{H}]$

Also, notice that the magnetic dipole at rest in an external (and homogeneous) electric field $\overrightarrow{\mathrm{E}}$ has momentum given by

$\overrightarrow{\mathrm{g}}=\frac{1}{\mathrm{c}^{2}}[\overrightarrow{\mathrm{M}} \times \overrightarrow{\mathrm{E}}]$

with $\overrightarrow{\mathrm{M}}$ denoting the density of magnetic moment. When the magnetism of the dipole changes, then the density of force is given by the last term of Eq.(11). 
Surprisingly, it can be shown that the Magnus force is analogous to the Abraham's force, as given by Eq.11. This similarity between vast areas of the physical theory, undoubtedly constitutes a successful example of the unity of physics, and illuminates the physical reality hidden inside the physical vacuum.

In fact, in a previous paper on "fluidic electrodynamics" [22], we introduced a new approach to the realm of the electromagnetic fields, in terms of the potential functions $(\vec{A}, \phi)$ and their material derivative, as they emerge in quantum mechanics as more fundamental quantities than the ( $\vec{E}, \vec{B}$ ) fields, predicting certain quantum interference effects, like the Aharonov-Bohm (AB) effect and the single-leg electron interferometer effect, known as the Josephson effect.

The new methodology offered by the "fluidic electrodynamics" approach is a helpful guide to propulsion and energy engineering, avoiding more complicated procedures. There is fundamental reasons to attribute to the vector potential $\vec{A}$ the property of the velocity of a "fluid" embedded in the physical vacuum. To describe the inner nature of a so pervasive and evasive medium, be it a mechanical medium whose deformations correspond to the electromagnetic fields, or a locally preferred state of rest, is beyond our purpose now. Possibly this issue is related to the Graham and Lahoz experimental findings that "something in motion" is not duly took into account in our present theories of the physical universe. To describe the electromagnetic field it is necessary to define the electric field $\vec{E}(\vec{r}, t)$, the magnetic field $\vec{B}(\vec{r}, t)$; charge density $\rho(\vec{r}, t)$; and the charge velocity $\vec{v}(\vec{r}, t)$. However, it is more advantageous to associate the set of Maxwell's equations with the electromagnetic potentials $\vec{A}(\vec{r}, t)$ and $\phi(\vec{r}, t)$ through the relationships:

$\vec{E}(\vec{r}, t)=-\vec{\nabla} \phi-\frac{d \vec{A}}{d t}$

and as well $\vec{B}=[\vec{\nabla} \times \vec{A}]$.

Note that we introduced into Eq.14 the convective derivative $d / d t=\partial / \partial t+\vec{v} \cdot \vec{\nabla}$, instead of the Maxwell-Einstein operator $\partial / \partial t$ (see, Ref. [22] and references therein).

Table 1 gives the correspondence of field variables in electromagnetism and hydrodynamics. Notice, for example, that $\vec{B}$ is the analogue to the angular velocity instead of the vorticity.

Table 1. Correspondence of field variables in electromagnetism and hydrodynamics

\begin{tabular}{|c|c|}
\hline ELECTROMAGNETISM & HUDRODYNAMICS \\
\hline$q / \varepsilon_{0}$ & Kraftquelle-q \\
\hline Permeability of the vacuum: $\mu_{0}$ & Mass density: $\rho$ \\
\hline Electric potential: $\phi$ & Massic entalphy: $\pi / \rho$ \\
\hline Scalar potential: $\chi$ & Potential velocity: $\Phi$ \\
\hline Vector potential: $\vec{A}$ & Velocity (or hydrodynamic momentum): $\vec{u}$ \\
\hline Electric field: $\vec{E}$ & Lamb vector: $\vec{l}$ \\
\hline Magnetic field: $\vec{B}$ & Angular velocity: $2 \vec{\omega}$ \\
\hline$U(\vec{r}, t)$ & $p(\vec{r}, t)=\rho(\vec{r}, t) \Phi(\vec{r}, t)-\frac{\rho(\vec{r}, t)}{2} u(\vec{r}, t)^{2}$ \\
\hline Electric current: I & Circulation: $\Gamma$ \\
\hline $\begin{array}{l}\text { Electromotiveforce: } \\
\vec{E}=-\nabla \phi-\frac{\partial \vec{A}}{\partial t}+[\vec{v} \times \vec{B}]-\nabla(\vec{v} \cdot \vec{A})\end{array}$ & $\begin{array}{l}\text { Hydromotiveforce: } \\
\vec{E}_{H}=-\frac{\partial \vec{u}}{\partial t}-\nabla\left(\frac{p}{\rho}+\frac{u^{2}}{2}\right)-[\vec{\omega} \times \vec{u}]\end{array}$ \\
\hline
\end{tabular}






Figure 3. An expanding toroidal disk.

The analogy suggests a new process of propulsion using the Abraham's force to accelerate a plasma jet. As already referred above, in the natural world, fishes and birds propel themselves through a liquid medium by using their limbs to transfer momentum to the liquid via vortex structures. Their basic mechanism is to form a vortex structure (e.g.: Ref. [21]). Hence, next we will look at the mechanism that generates the Magnus force. First of all, we may remark that it is necessary to consume energy in order to progressively enlarge the vortex with a characteristic radial velocity (see Fig. 3). The toroidal structure is a vortex ring formed by a closed vortex tube of a given diameter (let us say $\chi$ ). As is well-known in fluid dynamics, this structure is very stable. The duty mechanism that provides this radial velocity (inward or outward from the central axis) may have different sources. One example is gas falling into stars, in the case of polar jets; another is the sharp increase of electric current generated by the growing magnetic field of the plasma. With this kind of mechanism, we can associate a given circulation $\Gamma^{\prime}$ (due eventually to an induced field $\left.\omega^{\prime}\right)$. The falling (or expelled) stream of particles, most probably will acquire a curved trajectory and angular momentum, all effects concurring to the formation of the ring with circulation $\Gamma$ (and vorticity $\omega$ ). At the core of the vortex structure, the resultant force is aligned along the $Z$ axis. Newton's third law predicts a mechanical reaction force $\vec{F}_{m e c}$, which can propel a device (or a magnetized fluid). Therefore, we must have (in Figure 3, it represents the mechanical force pointing downward). This is what must happens with a fluid.

But what could be the analogue in the physical vacuum?

It can be shown that there is an electrodynamical counterpart - the Abraham's force - which plays an analogous in the formation of what we will call the "electromagnetoroid". Let us now explore the concept in more detail. Firstly, replace the "hydrodynamic magnetization" term in Eq.13 with the constitutive relationship $\vec{M}=\eta \vec{\omega}$, where $\eta$ represents a given property of the medium (a dimensionless constant). The outlined mapping shown in Table 1 gives us the analogous hydrodynamic force (by unit of length):

$d \vec{F}_{m}^{H}=-\frac{\rho}{c^{2}} \frac{\partial}{\partial t}[\vec{\omega} \times \vec{l}] d v$

Here, $\rho$ is the mass density and $d v$ is the differential volume element. Eq.(15) represents the interaction of physical entities fed by different energy sources: the circulation is associated with motion around the vortex-ring, while the Lamb vector is associated with the increasing vortex radius. The axial vector $\omega$ spirals about the azimuthal direction, forming a closed circular loop around the main axis.

It is interesting to note that Eq.(15) points to the existence of dual forces: one dependent on the fluid angular acceleration (or time-dependent magnetic force), the other dependent on the Lamb-vector time dependency (or time-dependent electric field).

Let us use the cylindrical geometry, shown in Fig.7, considering $\vec{\omega}=\omega_{\theta} \vec{u}_{\theta}$ and $\vec{l}=l_{r} \vec{u}_{r}$. Then, the total force resulting from this geometry is given by the following expression:

$\vec{F}_{m}^{H}=\frac{\rho}{c^{2}} \iiint_{S^{\prime}} \omega_{\theta} \frac{\partial l_{r}}{\partial t} \vec{u}_{z} d r d z r d \theta$

We can arrange terms to obtain

$F_{m}^{H}=\rho \int \omega_{\theta} \int_{S^{\prime}}\left[\vec{\nabla} \times \vec{\omega}^{\prime}\right] d z r d \theta d r$

where we inserted the hydrodynamic form of Ampère's equation:

$\frac{\partial \vec{l}}{\partial t}=-c^{2}\left[\vec{\nabla} \times \vec{\omega}^{\prime}\right]$

$\omega^{\prime}$ represents a different (axial) vector (than $\omega$ ). In fact, it is the vorticity associated with the increasing Lamb vector. The vorticity vector is oriented along the radial axis and $c$ is a characteristic speed of the medium. Hence:

$F_{m}^{H}=-\rho \int \omega_{\theta} \int_{\gamma}(\vec{\omega} \cdot d \vec{p}) d r$ 
However, we also have $\int_{\gamma}\left(\vec{\omega}^{\prime} \cdot d \vec{p}\right)=v_{r}{ }^{\prime}$. We therefore obtain

$$
F_{m}^{H}=-\rho \int \omega_{\theta} v_{r}{ }^{\prime} d r=-\rho v_{r}^{\prime} \int \omega_{\theta} d r
$$

The last integral of Eq.(34) is the circulation (by unit of length). This result can be recast in the following final form:

$$
\vec{F}_{m}^{H}=\rho[\vec{v} \times \vec{\Gamma}]
$$

Eq.(21) shows that Abraham's force is the electromagnetic analogue of Magnus's force in hydrodynamics (by unit of length). Therefore, we conclude that the Abraham's force represents a kind of vortex structure formed in the physical vacuum. The associated reaction force can propel a material structure through space. From this general mechanism (see, e.g., [28,29]), we can envisage the engineering of novel mode of spaceship propulsion based on generating electromagnetic vortices [30], along with a new framework to develop high-current accelerators and thermonuclear devices.

\subsection{The Concept of Electromagneto-Toroidal Gravity Machine}

At first, we consider the gravitational theory for a quasi-static field. Eq.(6) becomes

$$
\operatorname{rot} K \approx-\left(4 \pi G / c^{2}\right) J_{g} \text {. }
$$

As the current of mass generated by the electromagnetic field can be expressed as $J_{g}=S / c^{2}=\varepsilon_{0} E \times B$, where $S$ is the Poynting's vector of electromagnetic field, we can replace $J_{g}$ by the electromagnetic momentum $J_{e}=\varepsilon_{0} E \times B$ in Eq.(22). Then we have

$$
\int_{C} K \cdot d s \approx \frac{4 \pi G}{c^{2}} \iint_{S} J_{e} \cdot d S .
$$

From the equation $E=V /(r \cdot \log (b / a))$, where $V$ is the applied voltage, and $a, b$ are the inner and outer radii of the cylinder shown in Fig.4, we can obtain[17]

$$
K \approx \frac{4 \pi \varepsilon_{0} G}{c^{2}} V \cdot B \text {, }
$$

if we suppose there is no field of co-gravitational field.



Figure.4 Generation of $K$ field by the electromagnetic fields inside the tube structure

We consider the torus-shaped structure composed of a co-axial condenser and coils curling around the outer surface of the torus shown as follows:

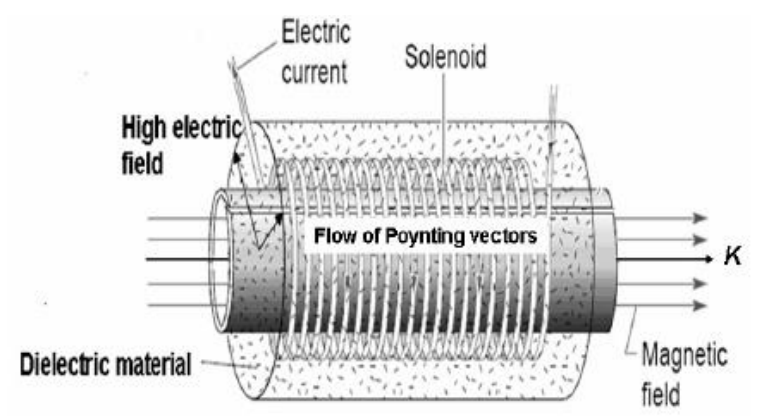

Figure.5 Schematic diagram to generate an impulsive electromagnetic field inside the Torus

A high intensity electromagnetic field can be generated by the structure, as shown in Fig.5.

As shown in this figure, an impulsive strong magnetic field is induced by an impulsive electric current through the coils embedded in the dielectric material under the intense electric field and a strong co-gravitational field is generated.

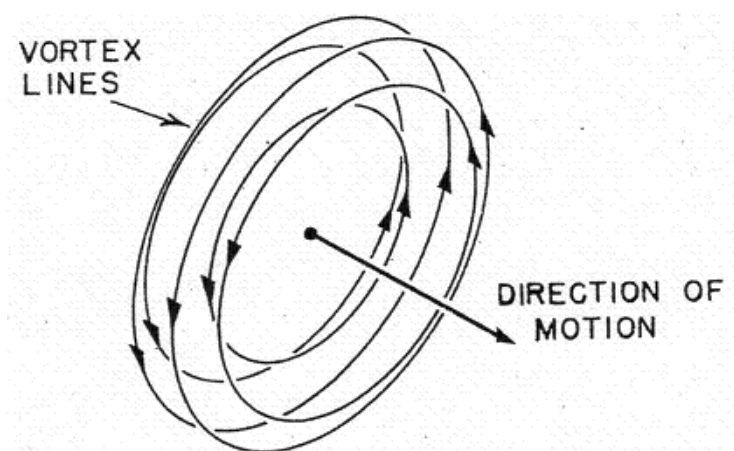

Figure.6 Moving vortex ring in a fluid

As the co-gravitational field is similar in scope to a vortex in fluid dynamics, as generated inside the 
torus shown in Fig.6, this torus-shaped bundle of vortex lines can cause forward motion of the ring, similar to the motion of a vortex within fluid dynamics.

As the electrical toroidal device is a kind of fluid accelerator, as shown in Ref. [18], acting on the material device with a density of force given by

$$
f \cong \frac{\varepsilon_{r} \mu_{r}}{c^{2}} \frac{\partial}{\partial t}[E \times H],
$$

we have

$$
f \cong \varepsilon_{r} \varepsilon_{0} \frac{\partial}{\partial t}[E \times B],
$$

which is shown in Fig.7 with its resulting direction. By corresponding electromagnetic and gravitational symbols and a constant given by $E \rightarrow g, B \rightarrow K$ and $\varepsilon_{0} \rightarrow-1 / 4 \pi G$, then it follows

$$
f \approx-\frac{1}{4 \pi G} \frac{\partial}{\partial t}[g \times K] .
$$

Figure.7 The electromagnetic generated force, analogous to the fluid dynamics

When we let $g=r \omega^{2}$ ( $r$; radius of the toroidal structure, $\omega$ : radial frequency of the spinning structure), we have

$$
|f| \approx \frac{\varepsilon_{0}}{c^{2}} r \omega^{2}\left(V \frac{\partial}{\partial t} B+B \frac{\partial}{\partial t} V\right),
$$

If we assume to simplify a uniform magnetic field, $B=$ const, we obtain the following order of magnitude

$$
|f| \approx 9.8 \times 10^{-29} \frac{v^{2}}{r} B \frac{\partial}{\partial t} V,
$$

where $v$ is the speed of the rotating toroidal structure. Therefore, from the above considerations, we may conclude that there is the possibility to generate a new gravitational field by applying impulsive high electromagnetic field to the toroidal device.

\section{Numerical Calculation for the Rotating Electromagneto- Toroidal Gravity Machine}

If the considered gravity machine could be operating with a velocity of the rotating toroidal structure to be one percent of light speed, and considering the parameters $r=5 m, \mathrm{~B}=100$ Tesla, $V=1$ Giga volt, and the duration of time for applying the impulsive high voltage electric field be 1.0 pico second, we have $f=1.8 \times 10^{7} \mathrm{~N}$, : according to Eq.(29), which is sufficiently large for thrusting a spacecraft. Hence, it is considered possible to provide a design that can allow the construction of an antigravity machine as follows:

It is composed of a co-axial condenser whose surface is overwound by the superconductor coil, which generates a gravitational vortex around the cylindrical body. Instead of Forward's gravity machine which utilizes the circulation of a ultra-dense, superfluid through a spiral tubing array, this system uses only a high intensity electromagnetic field generated by the co-axial condenser and coils to create a similar gravitational vortex.

Murad pointed out in his papers that Jefimenko's co-gravitational field is the elusive spin or torsion field identified in Russian scientific literature [31,32]. This idea was first introduced by the French mathematician Elie Cartan in 1913, then by Albert Einstein aiming to a unified theory. Within the framework of Cartan-Einstein theory, the existence of these fields has been permitted. Scientists today are recognizing that "spinning fields" really do exist. Just as electromagnetic fields are caused by a charge and gravitational fields are caused by weight, torsion fields are created by any rotating objects (see also $[33,34])$.



Figure.8 Rotating toroidal structure of the propulsion system

Figure. 8 shows the conceptual diagram of the gravity machine designed to propel the spaceship. By pulsing the electromagnetic field generated by the superconducting coil, a strong co-gravitational field can be generated inside the toroidal structure, as illustrated in Fig.8. When this toroidal structure is 
rotating at very high speed around the vertical axis, thrust can be generated to propel the spaceship.

\section{CONCLUSION}

In this work, some gravity machines, allowed within Einstein's general relativity theory, have been presented. One design worked using ultra-dense fluid matter, and another one utilizing an intense pulsing electromagnetic field. Through numerical calculation and quantitative discussion, the electromagneto-toroidal propulsion system can possibly produce sufficient thrust to propel a spaceship, similar to Forward's gravity machine. However, from an engineering perspective, the electromagneto-toroidal method seems to be far more practical. With further research into such new directions of space sciences, which are still nowadays based on conventional physics, we may, in the future, find a way to travel to the stars.

\section{References}

1. Crawford, I. A., Interstellar Travel: A Review for Astronomers, QJRAS, Vol. 31, 1990, pp.377-400

2. Hein, A.M., "Evaluation of Technological-Social and Political Projections for the Next 100-300 Years and the Implications for an Interstellar Mission". Journal of the British Interplanetary Society. 33 (09/10), 2012, pp. 330-340.

3. Long, Kelvin (2012). Deep Space Propulsion: A Roadmap to Interstellar Flight. Springer. ISBN 978-1-4614-0606-8.

4. Mallove, Eugene (1989). The Starflight Handbook. John Wiley \& Sons, Inc. ISBN 0-471-61912-4.

5. Forward, R.L., Antigravity, Proceeding of the IRE, 1961, pp.1442.

6. Forward, R.L., Guidelines to Antigravity, American Journal of Physics, 37, 1963, pp.166-170.

7. Woodward, James (2013). Making Starships and Stargates: The Science of Interstellar Transport and Absurdly Benign Wormholes. Springer. ISBN 978-1-4614-5622-3.

8. Zubrin, Robert (1999). Entering Space: Creating a Spacefaring Civilization. Tarcher / Putnam. ISBN 1-58542-036-0.

9. Harris E.G., Analogy between general relativity and electromagnetism for slowly moving particle in weak gravitational fields, American Journal of Physics, 59 (5), 1991,pp.421-425.

10. Braginsky, V.B., Caves, C.M., Thorne, K.S., Laboratory experiments to test relativistic gravity, Physical Review D, Vol.15, No.8, 1977, pp.2047-2068
11. Dirac. P.A.M., Is there an Aether?, Nature, vol.168, 1951, pp.906-907.

12. Dirac. P.A.M., Is there an Aether?, Nature, vol.169, 1952, pp.702.

13. Sinha, K.P., Sivaram, C., Sudarshan, E.C.G., Aether as a superfluid state of particle-antiparticle pairs, Found. Phys., Vol.6,No.1, 1976, pp.65-70.

14. Sinha, K.P., Sivaram, C., Sudarshan, E.C.G., The superfluid vacuum state, time-varying cosmological constant, and nonsingular cosmological models, Found. Phys., Vol.6,No.6, 1976, pp.717-726.

15. Sinha, K.P., Sudarshan, E.C.G., The superfluid as a source of all interactions, Found. Phys., vol.8, 1978, pp.823-831.

16. Musha, T., Possibility of Antigravity Propulsion by Gravitational Vortex, Proceedings of the 36th Conference on Aerospace Propulsion, JSASS, 1996, pp.321-326 (Japanese).

17. Pinheiro, M.J., Electromagnetotoroid Structure in Propulsion and Astrophysics, DFIST, 2009, pp.1-7.

18. Martins, A. A.; Pinheiro, M., Fluidic Electrodynamics: an approach to electromagnetic propulsion, J. Phys. Fluids 21, 2009, 097103.

19. Martins, A. A.; Pinheiro, M. J., The connection between inertial forces and the vector potential, AIP Conference Proceedings 880, 2007, 1189.

20. Feigel, A., Quantum Vacuum Contribution to the Momentum of Dielectric Media Phys. Rev. Lett. 92(2) , 2004, 020404-1.

21. Martins, A. A.; Pinheiro, M. J., On the electromagnetic origin of inertia and inertial mass, Int. J. Theo. Phys. 47 (10), 2008 , 2706-2715.

22. Harpaz, A.; Soker, N., Radiation from a Uniformly Accelerated Charge, Gen. Rel. Grav. 30 (8), 1998, 1217-1227.

23. Shockley, W.; James, R. P., A "Try Simplest Cases" Resolution of the Abraham Minkowsky Controversy on Electromagnetic Momentum in Matter, Science, 156 (3747), $1967,542$.

24. Huggins, Elisha, R., Exact Magnus-Force Formula for Three-Dimensional Fluid-Core Vortices, Phys. Rev. A 1 (2) 1970 , 327-331.

25. Brito, Hector H., Experimental status of thrusting by electromagnetic inertia manipulation, Acta Astronautica 54, 2004 , 547-558.

26. Cleveland, Blair, M., Electromagnetic propulsion via a vacuum-interactance push Electric Spacecraft 24, 1996, 6-16.

27. Pinheiro, Mario J., On Newton's third law and its symmetry-breaking effects, Physica Scripta 84 (5), 2011, 055004. 
28. Hedenström, Anders, A General Law for animal Locomotion, TRENDS in Ecology and Evolution 19(5) , 2004 , 217-219.

29. Robertson, G. A.; Pinheiro, M., Vortex Formation in the Wake of Dark Matter Propulsion, J. Physics Procedia, 20, 2011 , 6-23.

30. Murad, P.A., Its all gravity, Space Technology and Applications International Forum (STAIF-2003). AIP conference proceedings 654, NY, American Institute of Physics, 2003, pp.932-939.

31. Murad, P.A., A proposed model for capturing gravitational spin anomalies, Space Technology and Application International Forum (STAIF-2004), AIP conference proceedings 699, NY, American Institute of Physics, 2004, pp.1098-1105.

32. Pinheiro, Mario J., Some effects of topological torsion currents on spacecraft dynamics and the flyby anomaly, Monthly Notices of the Royal Astronomical Society, Volume 461, Issue 4, 1 October 2016, Pages 3948-3953

33. Pinheiro, Mario J., A reformulation of mechanics and electrodynamics, Heliyon 3 (2017) e00365 